well-preserved in the channel waveguides they fabricated, and believe that $\mathrm{O}^{3+}$ ion implanted channel waveguides in Nd:YAG ceramic are suitable as active integrated devices.

JOAN J. CARVAJAL

\section{Ab Initio Calculations Demonstrate Impermeability of Graphene with Defects to Helium Atoms}

Applications for graphene as ultrathin barrier membranes were envisaged after recent experiments showed that graphene sheets are impenetrable to standard gases, including He. It was also recently suggested that graphene needs to be free of defectseven single-atom vacancies-in order to maintain its impermeability. Recently, however, O. Leenaerts, B. Partoens, and F.M. Peeters from the University of Antwerp in Belgium used ab initio calculations to show that graphene with common defects is still impermeable, and that large defects are required to make graphene permeable.

As reported in the November 10, 2008 issue of Applied Physics Letters (DOI: 10.1063/1.3021413; \#193107), Leenaerts, Partoens, and Peeters used density functional theory (DFT) to simulate defects in a $4 \times 4 \times 4$ graphene supercell (one defect requires a larger supercell) with a distance of $16 \AA$ between adjacent layers. The researchers selected six defects that preserve the $s p^{2}$ hybridization of the 6-12 $\mathrm{C}$ atoms that form the periphery of the defect. The researchers first examined the approach of a He atom toward the center of a carbon hexagon in a graphene monolayer, keeping, as a first approximation, the $\mathrm{C}$ atoms fixed, and calculated the potential energy barrier to be so large that penetration at any temperature is impossible. The barrier to He penetration was barely decreased when the graphene was allowed to relax, as shown with molecular dynamics simulations performed within DFT formalism. The researchers showed that, at the turning point of the He atom, graphene relaxation is very small because it occurs on a much larger time scale than that for the motion of the much lighter He; almost all graphene relaxation occurs after deflection of the He atom. Graphene relaxation was therefore ignored to a very good approximation when calculating potential energy barriers to He penetration for graphene with defects, although the researchers said that this approximation may not hold for heavier penetrants.

For the six defects studied, the lowest penetration barrier was always found in the center of the defect, as expected intuitively. The researchers also found that the penetration barrier decreases exponentially with the size of the defect, as measured by the number of $\mathrm{C}$ atoms that form the defect; extrapolation shows that large defects are required for He penetration at room temperature. The researchers concluded that "even defective graphene is a suitable candidate for making impermeable nanomembranes for future applications and therefore can be considered the thinnest possible material for constructing a micro- or nanoballoon."

SteVEn TrohalaKi

\section{$\mathrm{Ho}^{3+}$-Doped Nanophase Glass Ceramics Enhance Efficiency of $\mathrm{Si}$ Solar Cells}

In the December 15, 2008, issue of Optics Letters (DOI: 10.1364/OL.33.002982; p. 2982), F. Lahoz from the University of La Laguna in Tenerife, Spain reports how $\mathrm{Ho}^{3+}$-doped oxyfluoride glass ceramics are suitable to up-convert photons from the $\sim 1170 \mathrm{~nm}$ sub-bandgap region of silicon into higher energy photons that can be absorbed by the silicon in solar cells. Since the proposed material is transparent at $1540 \mathrm{~nm}$, it can be used complementarily to the $\mathrm{Er}^{3+}$ phosphors that have also been proposed to increase the efficiency of solar cells.

In order to adapt the incident solar spectrum to the properties of commercially available solar cells fabricated with $\mathrm{Si}$, the use of photon upconversion materials is currently being investigated. Si is transparent to photons with wavelengths longer than its bandgap $(\sim 1.1 \mu \mathrm{m})$, and therefore the energy of these incident photons is lost. In an upconversion process, two or more low energy photons are transformed into one photon of higher energy. Therefore the upconversion material is able to absorb part of the subbandgap radiation and transform it into photons above the bandgap that can be absorbed by the solar cell and consequently create additional electron-hole pairs.

In Lahoz's work, efficient upconversion emission of $\mathrm{Ho}^{3+}$ ions in the visible $(\sim 650 \mathrm{~nm})$ and near-infrared $(\sim 910 \mathrm{~nm})$ regions is demonstrated when the sample is pumped at $\sim 1170 \mathrm{~nm}$. An oxyfluoride glass ceramic was selected as the host matrix. This material has a glassy structure with an oxyfluoride composition in which there are embedded fluoride nanocrystals. In order to produce the glass ceramic, the precursor glass underwent a thermal treatment $\left(470^{\circ} \mathrm{C}\right.$ for $\left.36 \mathrm{~h}\right)$. As a result, the fluoride nanocrystals $\left(\beta-\mathrm{PbF}_{2}\right)$ were precipitated and the $\mathrm{Ho}^{3+}$ ions partitioned into them. After processing, the photoluminescence intensity of the glass ceramic was two orders of magnitude larger than in the precursor glass.
Two main factors explain this increase. First, the $\mathrm{Ho}^{3+}$ ions in the glass ceramic are located in a favorable fluoride environment, which increases the probability of radiative transitions. Second, the distances between $\mathrm{Ho}^{3+}$ ions are reduced and this increases the probability of energy transfer among them and thus the probability of upconversion processes.

The researcher also discusses that the use of $\mathrm{Ho}^{3+}$ as a potential upconverter could be more efficient than that using the 1530$1560 \mathrm{~nm}$ absorption range ( $\mathrm{Er}^{3+}$ phosphors) since the solar irradiance at wavelengths near $\sim 1170 \mathrm{~nm}$ is about a factor of two more intense than in the former range. The researcher also said that both upconversion materials can be used simultaneously and proposes a double layer structure where first a $\mathrm{Ho}^{3+}$-doped glass ceramic is placed at the rear of a bifacial solar cell, followed by a second $\mathrm{Er}^{3+}$ phosphor with a mirror. This proposed structure offers the potential for an improvement of the efficiency of current Si solar cells.

Rosalía SERnA

\section{EXAFS Offers Evaluation of Intrinsic Template Binding Site of Molecularly Imprinted Polymers}

Molecular imprinting is a rapidly growing application which involves the design of molecularly imprinted polymers (MIPs) that possess recognition sites for molecules whose selectivity rivals that of natural antibodies. The binding cavities are fashioned through the extraction of the template molecule from the polymer, leaving behind a well-defined imprint cavity of specific structure and memory containing functional groups that enable specific, highaffinity molecular capture. Understanding these phenomena requires an efficient method to characterize the binding phenomena in a stable template cavity. To address this issue, C.J. Allender of Cardiff University, UK; S. Fiddy of CCLRC Daresbury, UK; M. Stockenhuber of the University of Newcastle, UK; and their colleagues have developed the first known direct experimental probe of a molecularly imprinted polymer active imprinting site using extended $x$-ray absorption fine structure (EXAFS), as reported in the January 8 issue of Chemical Communications (DOI: $10.1039 /$ b811578h; p. 165).

MIP's potential for enhanced stability, lower cost, and selectivity at lower concentrations in comparison to their natural derivatives make them very attractive materials. However, their widespread applicability is often hindered by issues such as slow and inefficient template binding kinetics. Using EXAFS, researchers have been able to define template-polymer 
interactions for a 4-vinyl pyridinestyrene-divinylbenzene copolymer imprinted with a dibenzoylmethane (DBM) and cobalt $\left(\mathrm{Co}^{2+}\right)$ system. EXAFS was successfully employed to determine the binding of the DBM in the polymer through identifying changes in the coordination shell of the cobalt.

Binding and template re-uptake studies with cobalt complexes using imprinted and non-imprinted polymer (NIP) controls provided variations in data fit to models with fixed coordination numbers. These models suggest structural differences in the cobalt species that is sequestered in the MIP in comparison to the NIP, as well as substantiate the type of cobalt complex bound to each polymer. These results were supported by UV-Vis and IR spectroscopic data.
The use of EXAFS by these researchers to probe the template binding site in a MIP provided insight into the binding site features. The success of this technique lends itself to application in many more MIP systems, with the possibility of eliciting an improved understanding of the scientific theories involved in creating and evaluating stable imprinted polymeric materials.

ANIKA A. ODUKALE

\section{Tough Ceramic Mimics Mother of Pearl}

Mother of pearl, or nacre, the inner lining of the shells of abalone, mussels, and certain other mollusks, is renowned for both its iridescent beauty and its amazing toughness. Nacre is 95\% aragonite, a hard but brittle calcium carbonate mineral, with the rest of it made up of soft organic molecules. Yet nacre can be 3000 times (in energy terms) more resistant to fracture than aragonite. No human-synthesized composite outperforms its constituent materials by such a wide margin. Now R. Ritchie and colleagues from Lawrence Berkeley National Laboratory have mimicked the structure of mother of pearl to create what may well be the toughest ceramic synthesized.

Through the controlled freezing of suspensions in water of an aluminum oxide (alumina) and the addition of polymethylmethacrylate (PMMA), the research team has produced ceramics that are 300 times tougher than their constituent components, as reported in the December 5, 2008 issue of Science (DOI: 10.1126/science.1164865; p. 1516).

Nacre's remarkable strength is derived from a structural architecture that varies over lengths of scale ranging from nanometers to micrometers. Human engineering has not been able to replicate these length scale variances. Two years ago, however, Berkeley Lab researchers A. Tomsia and E. Saiz found a way to improve the strength of bone substitutes through a processing technique that involved the freezing of seawater. This process yielded a ceramic that was four times stronger than artificial bone. When seawater freezes, ice crystals form a scaffolding of thin layers. These layers are pure ice because during their formation impurities, such as salt and microorganisms, are expelled and entrapped in the space between the layers. The resulting architecture roughly resembles that of nacre.

"Since seawater can freeze like a layered material, we allowed nature to guide the process by which we were able to freeze-cast ceramics that mimicked nacre," said Tomsia when this research was reported.

In this latest research, Ritchie, working with Tomsia and Saiz, refined the freeze-casting technique and applied it to alumina/PMMA hybrid materials to create large porous ceramic scaffolds that much more closely mirrored the complex hierarchical microstructure of nacre. To do this, they first employed directional freezing to promote the formation of thin layers (lamellae) of ice that served as templates for the creation of the layered alumina scaffolds. After the ice was removed, spaces between the alumina lamellae were filled with polymer.

"The key to material toughness is the ability to dissipate strain energy," said Ritchie. "Infiltrating the spaces between the alumina layers with polymer allows the hard alumina layers to slide (by a small amount) over one another when load is applied, thereby dissipating strain energy. The polymer acts as a lubricant, like the oil in an automobile engine."

In addition to making the lamellar scaffolds, the team was

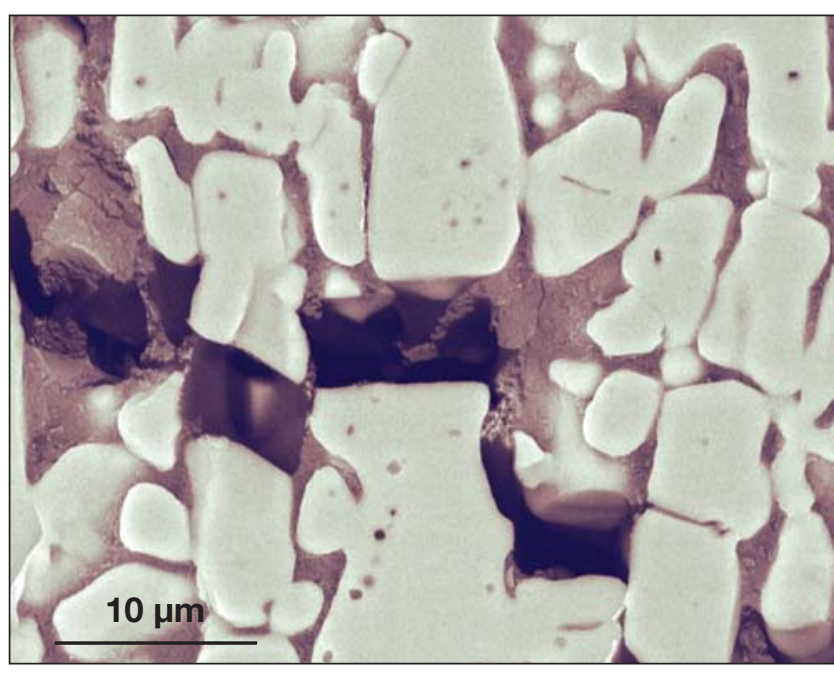

Figure 1. In the "brick-and-mortar" phase of the alumina/ PMMA hybrid, aragonite "bricks" slide past each other to dissipate strain energy while the polymer "mortar" acts as a lubricant.

also able to fabricate nacre-like "brick-and-mortar" structures with very high alumina content (see Figure 1). They did this by collapsing the scaffolds in a perpendicular direction to the layers, then sintering the resulting alumina "bricks" to promote brick densification and the formation of ceramic bridges between individual bricks.

Saiz said, "Using such techniques, we have made complex hierarchical architectures where we can refine the lamellae thickness, control their macroscopic orientation, manipulate the chemistry and roughness of the inter-lamellae interfaces, and generate a given density of inorganic bridges, all over a range of size-scales."

For ceramic materials that are even tougher in the future Ritchie said he and his colleagues need to improve the proportion of ceramic to polymer in their composites. The alumina/ PMMA hybrid was only $85 \%$ alumina. They want to boost ceramic content and thin the layers even further. They also want to replace the PMMA with a better polymer and eventually replace the polymer content altogether with metal.

Ritchie said, "The polymer is only capable of allowing things to slide past one another, not bear any load. Infiltrating the ceramic layers with metals would give us a lubricant that can also bear some of the load. This would improve strength as well as toughness of the composite."

Such future composite materials would be lightweight and strong as well as tough, he said, and could find important applications in energy and transportation. 